\title{
Lútopie de Thomas More: Une réponse au débat sur le Nouveau Monde?
}

Strosetzki, Christoph

First published in:

Moreana, 27. Jg., Heft 101-102, S. 5 - 24, Angers 1990 


\title{
L'Utopie de Thomas More: UNE Réponse aU oébat sur le Nouveau Monde?
}

\begin{abstract}
e débat sur l'utopie n'a jamais cessé d'être d'actualité. ${ }^{1}$
De tous temps, l'influence de Thomas More a été sensible en Amérique Latine. Il est, par exemple, l'un des modèles du mexicain José Joaquín Fernández de Lizardi pour El periquillo Sarniento (1819), un des tout premiers romans et la première utopie littéraire d'Amérique Latine. Et on retrouve fréquemment dans les romans latino-américains contemporains l'empreinte de l'Utopie. ${ }^{2}$

Mais, du vivant de Thomas More, l'oeuvre avait déjà d'importantes répercussions sur la réalité politique américaine. Ainsi, il semble bien qu'elle ait influencé Bartolomé de Las Casas (1474-1566), l'inlassable défenseur des Indiens : en 1515, Las Casas avait présenté à la Cour d'Espagne diverses propositions pour l'organisation du Nouveau Monde. Il est vraisemblable que l'Utopie joua un rôle dans la révision de ces textes avant leur présentation à Charles Quint, en $1517 .{ }^{3}$

De même, tandis que Las Casas essayait vainement, entre 1537 et 1550 , d'imposer ses idées à Verapaz (Guatemala), Vasco de Quiroga (1470-1565) installait ses propres "Hospitales" à Mexico dès 1531, puis à Michoacán (dont il deviendra l'évêque en 1537). Quiroga y appliquait le modèle défini par Thomas More. D'ailleurs, les "Ordenanzas" (1565) qui régissaient les "Hospitales" rappellent étonnamment les villes de l'Utopie. Dans son testament, Quiroga eut, par ailleurs, soin d'exiger que I'on se conformât toujours à ses ordonnances : cela explique qu'aujourd'hui encore son influence semble persister. ${ }^{4}$

Quant à "l'Etat jésuite" du Paraguay, il connut un grand retentissement, en dépit des controverses, ${ }^{5}$ et il fut perçu comme une utopie bâtie sur le modèle de Thomas More ${ }^{6}$ : de 1610 à $1768,160.000$ Indiens se répartissaient 30 districts sans propriété privée et dans le respect de la liberté personnelle.
\end{abstract}


De nombreux travaux attestent cette influence qu'a exercée l'Utopie sur le Nouveau Monde. En revanche, rares sont les recherches traitant de la source d'inspiration qu'a pu constituer le Nouveau Monde pour l'Utopie. Certes, on a bien souligné que Thomas More citait Améric Vespuce parmi ses influences. ${ }^{7 \& 8}$ Mais les chercheurs omettent généralement de rappeler que la découverte du Nouveau Monde soulevait, à l'époque, de nombreux problèmes philosophiques, juridiques et politiques dont on discutait beaucoup : le droit de prendre possession d'une terre étrangère, la christianisation, la cohabitation entre Espagnols et Indiens.

Dans quelle mesure Thomas More a-t-il eu connaissance de ces questions et a-t-il proposé, dans son Utopie, des solutions s'approchant de celles des théoriciens espagnols?

\section{Convergence de l'oeuvre de Thomas More et de la problématique du Nouveau Monde}

L'Utopie est divisée en deux parties. La première rappelle les désagréments de la vie en Europe et décrit les coutumes de pays lointains. On y apprend que Thomas More, envoyé en Flandres, écoute avec Pierre Gilles les récits d'un certain Raphaël Hythlodée qui aurait accompagné Amerigo Vespucci lors de ses voyages. Toutefois, l'analyse de son nom,

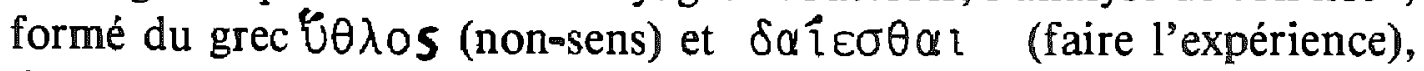
démontre qu'il s'agit là d'un personnage fictif.

Ce n'est qu'après son retour en Angleterre que More devait écrire cette première partie et la lettre adressée à Pierre Gilles. Quant à la seconde, c'est bien en Flandres, donc avant, qu'il la conçut. La présente recherche se limitera donc à la période qui se situe avant et pendant la mission de Thomas More en Flandres.

La découverte du Nouveau Monde posait, fondamentalement, une question : est-il légitime de coloniser et de christianiser ? Le sujet intéressait tout particulièrement les milieux juridiques. ${ }^{9}$ Il est probable que Thomas More ait déjà été confronté à ces questions lors de ses études de droit public à Lincoln's Inn ou à New Inn. ${ }^{10}$ Outre sa formation littéraire et ses activités d'Under-sheriff, il s'intéressait aux droits international et maritime. Il avait même appris le français pour mener à bien des tâches diplomatiques et prit part, en 1509, aux négociations entre les "Merchant Adventurers" et la ville d'Anvers. Tout cela démontre son goût pour le droit international. " Rappelons également qu'il avait 
décidé d'embrasser la carrière juridique sur les conseils de son père, luimême juriste.

Quant à son intérêt pour l'Amérique, il est probable que John Rastell, son beau-frère, ait contribué à l'éveiller, Rastell préparant sans doute déjà le voyage qui l'emmènerait vers les territoires vierges. ${ }^{12}$

Thomas More est donc envoyé en mission pour régler une question de droit international : de mai à octobre 1515, il est en Flandres pour modifier les tarifs douaniers frappant les Anglais. Ces droits de douane reposent sur deux traités ("Intercursus magnus" de 1496 et "Intercursus malus" de 1506). ${ }^{13}$ Les négociations entamées à Bruges sont bientôt bloquées. Les Flamands se retirent pour consultation auprès de Charles Quint. Thomas More en profite pour rendre visite à Pierre Gilles, à Anvers.

More ne participera pas à la rédaction finale du nouveau traité puisqu'il lui faudra rentrer en Angleterre, mais il a eu le temps de rencontrer Erasme à Bruges et Busleiden à Malines. ${ }^{14}$

More décrivit la rencontre avec Pierre Gilles (1486-1533) comme l'événement le plus agréable de son séjour en Flandres, ${ }^{15}$ à tel point que c'est à lui qu'il dédia l'Utopie. Gilles participe au dialogue dans le jardin de l'hôtel de More à Anvers. On peut donc raisonnablement imaginer que les deux hommes discutèrent largement de la deuxième partie de l'Utopie.

En ce qui concerne Pierre Gilles, rappelons qu'après avoir travaillé chez l'imprimeur anversois Dirk Martens d'Alost, il avait repris ses études de droit, sur les conseils d'Erasme. D'abord jurisconsulte, il devint, en 1509, greffier de la ville d'Anvers. On pouvait rencontrer chez lui, non seulement Erasme ou Vivès, mais également la plupart des célébrités de l'époque. De telle sorte que Thomas More put, auprès de lui, facilement s'informer des problèmes qui préoccupaient les savants du continent.

Toujours à propos de Gilles, rappelons qu'il est l'auteur d'un ouvrage d'histoire du droit, paru en 1517 , sur lequel il travaillait certainement lors de la visite de More. Il s'agit d'une compilation de lois romaines en usage chez les Wisigoths, qu'il considérait comme la source du droit justinien. ${ }^{16} \mathrm{Il}$ est probable que ces travaux intéressèrent Thomas More qui, Jui aussi, cherchait des lois pour son organisation utopique.

C'est sans doute chez Pierre Gilles que More entendit parler de l'humaniste espagnol Juan Luis Vivès et qu'il fit sa connaissance lors de sa visite diplomatique. ${ }^{17}$ Vivès (1492-1540), à cause de son sang juif, vivait loin de sa Valence natale. Après avoir étudié la philosophie scholastique à Paris, il s'était installé à Bruges, en 1512, où il établit des liens 
amicaux avec toute la colonie espagnole. De 1519 à 1522 , il fut professeur au "collège trilingue" de l'université de Louvain. Il y travailla, en particulier, avec Adrien d'Utrecht, le futur pape Adrien VI qui étudia, sans aucun doute, les fameuses propositions de Las Casas, avant même leur publication en 1516. D'autre part, Vivès fut, un certain temps, précepteur du jeune Guillaume de Croy, le futur archevêque de Tolède, et entra ainsi à la cour de Charles Quint. Quand on sait qu'en outre, ce jeune homme était le neveu du très influent Croy auquel More s'opposa durant sa mission, 18 il ne fait plus de doute que Thomas More discuta avec Vivès et en reçut, directement ou indirectement, de précieuses informations sur la problé-matique de l'Espagne et du Nouveau Monde.

Vieil ami de Thomas More, Erasme, lui aussi, entretenait des relations intenses avec l'Espagne. Célèbre grâce à son édition critique du Nouveau Testament (1516), il avait même été invité par le cardinal Cisneros à collaborer à la Bible Polyglotte qui était en chantier à l'université d'Alcalá. 19

Quant à Jérôme Busleiden (1470-1517), ses liens avec l'Espagne sont notoires. Théologien et juriste, il avait accompli d'importantes missions diplomatiques à Rome, en France et en Angleterre avant de se voir confier les préparatifs du premier voyage de Charles Quint vers l'Espagne. Il mourut, en août, à Bordeaux avant d'avoir atteint la frontière. Toutefois, grâce à sa longue expérience, il devait déjà avoir une grande connaissance de l'Espagne lorsque More lui rendit, en 1515, cette visite si agréable, tant Jérôme Busleiden était un personnage riche et généreux. ${ }^{20}$

En résumé, on peut affirmer que, lors de son voyage en Flandres, et même avant, Thomas More a eu tout loisir de se familiariser avec la problématique de l'Espagne et du Nouveau Monde, et qu'il n'a certainement pas manqué de le faire, étant donné son intérêt pour le droit international et la politique étrangère.

Quels étaient donc ces problèmes qui se posaient entre l'Espagne et le Nouveau Monde?

\section{La problématique du Nonveau Monde}

Les Espagnols avaient découvert une terre nouvelle occupée par des indigènes. Ils en avaient pris possession, souvent par la force. Il leur fallait ensuite installer un régime gouvernemental. Mais un certain nombre d'opposants posèrent alors les questions suivantes : Cette prise de possession est-elle légitime ? L'usage de la force est-il légitime ? Quelle forme donner à la nouvelle coexistence au sein de l'Etat ? 
Selon la tradition médiévale, quiconque découvrait une terre déserte pouvait en prendre possession. Mais il existait d'autres façons d'acquérir un territoire étranger : le mariage, l'héritage, le pacte, l'ordre du roi ou l'ordre du pape. C'est ainsi qu'en 1493, le pape avait ordonné aux Rois Catholiques de propager la foi dans les terres qu'ils venaient de découvrir, en délimitant les zones d'influence portugaise et espagnole. De là, la conviction que le pape avait fait des souverains catholiques ses délégués, ses vicaires responsables de la christianisation. ${ }^{21}$ De là, également, le système de l'encomienda, cet univers où les indigènes devaient vivre sous la tutelle des Espagnols. Grâce à cette coexistence forcée, la conversion des Indiens devait se faire plus facilement et il devait être plus facile de les faire travailler. C'est pourquoi les Rois Catholiques interdirent d'isoler les Indiens et encouragèrent les mariages interraciaux. ${ }^{22}$

Le commerce des biens fut, lui aussi, soumis à réglementation, et on érigea la fameuse casa de contratación de Séville. Désormais, il fut impossible d'échanger les trésors indiens contre de la pacotille ou de fournir des armes aux indigènes. Dans le même ordre d'idée, la vente d'esclaves indiens fut abolie, toutes ces mesures ayant caractère rétroactif. ${ }^{23}$

Pourtant des Indiens se soulevèrent contre le système de l'encomienda, et l'on fit parler les armes. Aussitôt, on se mit à discuter du procédé : dès 1510, Johannes Maior prôna à Paris la légitimité de la force afin de conduire plus vite les Indiens à la conversion. Mais ce débat n'atteignit son apogée qu'en 1550, quand Ginés de Sepúlveda s'opposa à Las Casas. Pour Sepúlveda, qui s'appuyait sur Aristote, les peuplades incultes devaient servir les peuples doués de raison. L'argument n'était pas nouveau puisque, dès 1511, le dominicain Antonio de Montesinos y avait répondu en déclarant, à Saint-Domingue, que les Indiens étaient, eux aussi, des êtres humains doués de raison. Cette prise de position fut d'ailleurs à l'origine de l'action de Las Casas en faveur des indigènes. ${ }^{24}$

Mais on considérait alors que des peuples s'adonnant au sacrifice humain ne pouvaient être doués de raison. Et les Rois Catholiques ordonnèrent, en 1503, de réduire en esclavage les cannibales, qui non seulement contrariaient l'action des missionnaires, mais s'attaquaient en plus aux indigènes déjà soumis. Tel fut, par exemple, le cas des Caraỉbes. ${ }^{25}$ Bref, la guerre servait la christianisation, mais permettait aussi de punir le crime. Autrement dit, pour justifier leur action, les Espagnols s'en tenaient à leur seul jugement à l'égard des Indiens.

C'est dans ce cadre que s'inscrivent les encomiendas, lancées par les ordonnances royales du 16 septembre 1501 adressées au gouverneur Ovando. L'ordre était de convertir les Indiens. Les moyens étaient, en 
théorie, la bienveillance et la protection. Se marier avec une Indienne n'était possible qu'avec le consentement de la jeune fille. "Como nuestros buenos súbditos y vasallos", ${ }^{26}$ l'Indien était soumis à l'impôt mais ne pouvait être contraint à travailler qu'en échange d'un salaire. En 1503, des décrets complémentaires ordonnèrent la construction d'une église et l'affectation d'un prêtre par pueblo. A côté de l'église, une école permettrait d'enseigner à lire et à écrire. Le prêtre se chargerait également d'apprendre aux Indiens qu'une partie de leurs ressources appartenait à l'Eglise et une autre à la Couronne ; que, comme les Espagnols, ils devaient vivre dans une maison avec leur famille, cultiver la terre et élever leur bétail, qu'il ne fallait en aucun cas s'emparer du bien d'autrui, etc. Pour appliquer cette loi, un responsable serait nommé. Les indigènes abandonneraient certaines coutumes. On établirait des dispensaires, etc. ${ }^{27}$

La répartition des Indiens (repartimiento) s'acheva en 1509. Chaque dignitaire espagnol reçut la charge de cent, quatre-vingts, soixante ou trente Indiens. Il semble, pourtant, qu'on ne respecta pas cette division : dès 1512, un texte établit que le maximum était de 300 Indiens, chiffre au delà duquel tout enseignement religieux était impossible. ${ }^{28}$

Bien que les décrets royaux fussent particulièrement laxistes, donc mal respectés, le sermon de Montesinos déjà mentionné fit un véritable scandale et attira l'attention du roi. Considérant que le dominicain était indirectement responsable de l'insurrection des Indiens, Ferdinand fit réprimander Montesinos par son Conseil Provincial. Traduit devant le souverain, Montesinos devait pourtant obtenir qu'une commission, réunie à Burgos en 1512, discutât des questions soulevées par la situation en Amérique. Le résultat de ces débats entre théologiens et juristes, connu sous le nom de Leyes de Burgos de 1513, fut une rationalisation des textes existants, le maintien de l'encomienda et une redéfinition des droits et devoirs respectifs des Indiens et des encomenderos.

Ainsi, l'encomendero devait, pour ses cinquante Indiens, construire quatre huttes de taille réglementaire. Il devait mettre à leur disposition des terres suffisantes pour la culture du maïs et l'élevage, ladite terre restant propriété des Indiens, même en cas de changement de maître. L'encomendero devait construire une église, et prier avec ses Indiens matin et soir. Tous les enfants devaient être baptisés dans les huit jours suivant leur naissance et recevoir un enseignement religieux, soumis à examen tous les quinze jours. Dans chaque groupe, l'encomendero pouvait désigner un individu chargé d'apprendre à lire et à écrire. Les Franciscains prenaient les fils de caciques en charge dès leur treizième année. 
Dans le même temps, les Indiens pouvaient revendiquer un salaire convenable, une nourriture suffisante comprenant au moins un plat de viande le dimanche, et une somme annuelle pour se vêtir. Les travailleurs des mines avaient, en outre, droit à une nourriture plus consistante et à quarante jours de congé tous les cinq mois afin de recevoir un enseignement religieux. En 1513, un amendement promulgué à Valladolid établit qu'après une période de neuf mois de travail, tout paysan pouvait s'adonner à la culture de ses propres terres. ${ }^{29}$

Quant au respect de la loi, il était contrôlé par des visitadores choisis parmi les colons espagnols les plus âgés qui, tous les deux ans, devaient rendre compte de la situation. Ces mêmes visitadores étaient soumis au contrôle d'un juge d'instruction dépendant du gouverneur. Cela devait permettre, en particulier, de vérifier qu'aucun encomendero n'employait d'Indien étranger à sa concession. ${ }^{30}$ Mais la justification profonde de toutes ces mesures est dans l'idée que le roi Ferdinand se faisait des Indiens : il les croyait paresseux et vicieux ${ }^{31}$ et pensait que, lorsqu'ils quittaient leur maître, "olvidan luego todo lo que les han enseñado y tornan a su acostumbrada ociosidad y vicios ". 32

Il n'était pas facile pour les encomenderos de conjuguer le travail obligatoire et la liberté des Indiens. C'est d'ailleurs ce qui explique cet argument de l'évêque Bernardo de Mesa selon lequel les Indiens, étant trop pauvres pour payer leur tribut, devaient s'en acquitter par le travail. Certains se posèrent donc la question de la durée de cette dépendance, qui était à mi-chemin entre la sujétion politique et l'esclavage transmis par voie d'hérédité. Pour y répondre, on en appela à Aristote, à Thomas d'Aquin mais également à Duns Scot pour qui cette domination était pleinement justifiée du fait que la liberté conduisait les barbares au vice. Ainsi, tandis que les Leyes de Burgos tendaient à une sujétion de type féodal, la réalité se rapprochait davantage de l'esclavage pur et simple. C'est en tout cas ce qu'indiquent les multiples protestations adressées par les dominicains à la Cour entre 1515 et $1518 .{ }^{33}$

Les problèmes du Nouveau Monde étaient donc nombreux : juridiques, théologiques et sociaux. Mais l'intérêt qu'il suscitait était également inspiré par la recherche de l'or. Même Christophe Colomb avait espéré en rapporter. Aussi, lorsque se répandit la rumeur que l'on avait trouvé de nouvelles mines, on assista à une véritable ruée ${ }^{34}$ et l'on envoya les Indiens arracher à la terre le précieux minéral. Les Leyes de Burgos elles-mêmes établissaient que tout encomendero devait employer dans les mines un tiers de ses Indiens. Paradoxalement, c'est à cette époque-là que renaît le mythe de l'Age d'Or, étayé sans doute par 
l'absence de propriété privée. Pedro Mártir de Anglería écrivit même que les habitants de Saint-Domingue étaient heureux parce qu'ils vivaient nus et sans argent: "Es cosa averiguada que aquellos indigenos poseen en común la tierra, como la luz del sol y como el agua, y que desconocen las palabras tuyo y mío, semillero de todos los males. Hasta tal punto se contentan con poco, que en la comarca que viven antes sobran campos que faltan a nadie. Viven en plena Edad de Oro, y no rodean sus propiedades con fosos, muros, ni setos". ${ }^{35}$ Et l'auteur de souligner que, pour ces gens-là, toute loi est inutile puisque leur instinct ne les porte qu'au bien. On retrouve la même idéalisation des Indiens dans les descriptions de Christophe Colomb, qui croyait avoir découvert un paradis terrestre. Non seulement le paysage frappait par sa luxuriance et son éternel printemps, mais les indigènes eux-mêmes étonnaient par leur beauté, leur gentillesse, leur simplicité et leur intérêt pour la religion chrétienne. Un certain León Pinelo tenta même de démontrer que le Paradis de la Bible se trouvait en Amérique.

Il n'est pas étonnant qu'un tel continent ait éveillé tant d'espoirs. D'une part, les gens cupides pouvaient rêver à la fortune ; d'autre part, les hommes d'église songeaient à la conversion des peuplades indigènes ; enfin, les politiques entrevoyaient la possibilité de construire une société nouvelle, fondée sur la raison, le savoir et une harmonieuse cohabitation. ${ }^{36}$ Tout cela explique que des humanistes comme Thomas More aient pu s'intéresser aux problèmes de cette coexistence et reprendre la réflexion de Platon sur la République idéale.

\section{Les solutions proposées par l'Utopie}

Les rapports entre l'Utopie et le Nouveau Monde sont de deux sortes : d'une part, les similitudes entre l'oeuvre et la réalité des Amériques, et d'autre part, certains éléments de l'oeuvre qui pourraient être des essais de solution idéale et spéculative.

Il est difficile de faire la part des choses et de dire si telle image est issue de la tradition littéraire et philosophique ou bien des informations sur le Nouveau Monde, d'autant que les deux sources se sont souvent combinées.

Nous allons donc traiter quelques exemples significatifs de convergence entre le Nouveau Monde et l'Utopie.

L'île d'Utopie, auparavant appelée Abraxa, doit son nom à Utopos, qui lui a apporté une culture et des usages nouveaux. Après avoir commandé à ses soldats et aux indigènes de creuser un isthme pour sépa- 
rer définitivement l'île du continent et ainsi mieux la protéger, le colonisateur planifie l'installation et l'organisation des agglomérations : cinquante quatre villes partageant la même langue, les mêmes institutions, les mêmes lois. ${ }^{37}$

Utopie nous est donc présentée comme le fruit d'un projet aussi vaste que rationnel. Pourtant, Thomas More est conscient des problèmes que pose le passage de la théorie à la pratique. C'est pourquoi il insiste particulièrement sur la nécessité des compromis et des moyens détournés. ${ }^{38}$

De même, il s'interroge sur le bien-fondé de la prise de possession du territoire et sur la légitimité de la guerre. Chez les Utopiens, la fondation de nouvelles colonies s'impose si leur population augmente notablement. Dans ce cas, ils font en sorte que leurs lois prévalent sur le nouveau territoire et tentent d'intégrer les indigènes afin que tous jouissent des mêmes lois et tirent le meilleur parti de la terre. Quiconque refuse cette intégration est chassé du pays, au besoin par la force des armes. Ainsi est-il juste de faire la guerre contre ceux qui ne tirent aucun profit de leurs terres, les possèdent donc sans justification et empêchent par là même d'autres individus d'exercer leur droit naturel à la propriété et à la nourriture. ${ }^{39}$ Apparemment, les Utopiens espèrent, de la part des indigènes colonisés, l'accueil chaleureux qu'ils réservent eux-mêmes aux étrangers. 40

Pour les Utopiens, la guerre et la gloire militaire ne sont rien. C'est pourquoi, lorsqu'un conflit est inévitable, ils recrutent des mercenaires. Il s'agit, en particulier, de sauvages Zapolètes qui vivent dans des forêts ou dans le désert et s'attaquent tantôt à leurs voisins, tantôt à leurs propres congénères. Dans l'esprit des Utopiens, il est légitime de faire la guerre pour défendre ses frontières ou celles de ses alliés, ou bien pour libérer un peuple opprimé. Il est également légitime d'attaquer pour punir une injustice ; par exemple, lorsque des marchands alliés sont lâchement malmenés. Quand les Utopiens eux-mêmes subissent un préjudice sans qu'il y ait atteinte à leur intégrité physique, ils se contentent de rompre leurs relations commerciales avec l'autre partie. Mais s'il y a sévices, ils déclarent la guerre, à moins qu'on ne leur livre le coupable. ${ }^{41}$

Quant à la christianisation, par laquelle les Espagnols légitimaient leur action, les Utopiens y ont trouvé une solution. La religion peut être différente, non seulement d'une ville à l'autre, mais également à l'intérieur d'une même ville. Toutefois, et comme tout le monde s'accorde sur l'existence d'un être suprême, dès que les indigènes entendent parler de Jésus, de sa doctrine, de sa vie et de ses martyrs, un bon nombre 
s'empresse d'embrasser le christianisme. Le narrateur estime que cette conversion peut s'expliquer soit par la similitude entre la religion indigène et le christianisme, soit par une grâce divine, soit enfin parce que le communisme plaisait au Christ, et est pratiqué par ses meilleurs disciples. A ce propos, on ne saurait passer sous silence l'ironie dont fait preuve le narrateur lorsqu'il signale qu'il n'y a pas de prêtres pour dispenser tous les sacrements nécessaires : c'est plutôt l'inverse qui se produisait en Amérique.

Les indigènes d'Utopie nouvellement convertis ne sont jamais molestés par les autres. Ce qui est bien sûr "utopique", quand on songe aux sévices infligés par leurs compatriotes aux convertis américains. Toutefois, la tolérance a ses limites en Utopie : on est obligé d'y croire qu'après sa mort, l'homme est puni ou récompensé selon ses actes. ${ }^{42}$

L'Utopie ne manque pas non plus d'aborder une autre grande question : les Utopiens pratiquent l'esclavage. Mais les esclaves, chargés des travaux pénibles ou dégradants, ne sont pas en général des prisonniers de guerre. Il s'agit de criminels utopiens, d'étrangers condamnés à mort et exilés ou encore de journaliers étrangers qui ont préféré l'esclavage en Utopie plutôt que la misère ailleurs. Ces derniers sont d'ailleurs considérés presque comme des citoyens à part entière. ${ }^{43}$

D'une manière générale, la hiérarchie est fondée sur la confiance en l'autorité supérieure : le phylarque dirige trente foyers ; dix phylarques et leurs foyers respectifs élisent un protophylarque, ainsi que le prince ; les fonctionnaires reçoivent le titre de "Père", etc. ${ }^{44} \mathrm{De}$ telle sorte que le principe féodal appliqué en Amérique est, ici, complété par un système électoral. Remarquons au passage que le phylarque a essentiellement pour tâche de veiller à ce que personne ne se laisse aller à la paresse ${ }^{45}$, ce qui en fait l'exact pendant de l'encomendero du Nouveau Monde.

A l'instar de l'encomienda, on attribue à chaque famille utopienne des terres qu'elle est chargée d'exploiter. Quelques citoyens sont toutefois dispensés de travail pour pouvoir se consacrer entièrement à leurs études. Comme dans l'encomienda, les prêtres sont chargés de l'éducation des enfants. Très considérés, ils exercent leur sacerdoce dans de superbes sanctuaires qui n'excluent aucune religion. ${ }^{46}$ Toutefois, à la différence de l'encomienda, où les prêtres devaient s'efforcer d'inculquer aux Indiens les rudiments de l'écriture et de la foi, les indigènes d'Utopie sont des prodiges d'intelligence, puisqu'en trois ans à peine, ils sont capables de lire n'importe quel auteur grec dans le texte. Ce qui permet au narrateur d'avancer que les Utopiens descendent des Grecs. D'autant 
qu'eux-mêmes lisent sans peine Platon, Aristote, Théophraste, Plutarque, Homère, etc. Mais ils disposent de l'imprimerie, et peuvent ainsi mieux divulguer ces auteurs. Peut-être peut-on considérer cela comme une idéalisation, très libre, de la situation que les Européens auraient aimé trouver dans le Nouveau Monde. ${ }^{47}$

Comme l'encomienda, Utopie jouit de lois et de règles dont le but est d'éviter que les autorités n'abusent de leur pouvoir. On y a, par exemple, prévu une alternance entre les travaux des villes et les travaux des champs (alternance de deux ans en Utopie). La journée de travail ne doit en aucun cas dépasser six heures, et elle est complétée par un imposant programme culturel, 48

A l'instar de l'encomienda, le ravitaillement est centralisé : chaque ville est divisée en quatre quartiers, lesquels entreposent les denrées que l'aîné de chaque famille vient retirer gratuitement. Ces mêmes entrepôts abritent les biens nécessaires aux banquets communautaires pris dans de grands réfectoires. Quant aux malades, ils sont l'objet d'une attention toute particulière : comme dans le Nouveau Monde, ils sont accueillis dans des hôpitaux. ${ }^{49}$

Mais tous ces droits sont assortis de devoirs. Un Utopien veut-il entreprendre un voyage ? Il doit en demander la permission au phylarque ou au protophylarque, et, une fois sur place, s'adonner aux travaux qu'on lui confiera, afin de prévenir toute tendance à l'oisiveté. De même, tout abandon de poste est sévèrement puni. ${ }^{50} \mathrm{Bref}$, les Utopiens ont un sort qui rappelle étrangement celui des Indiens des encomiendas.

Pour les Utopiens, la vertu revient à vivre en conformité avec la nature. Ils n'accordent de valeur ni aux vêtements, ni aux honneurs, ni au jeu, ni aux biens. Seules importent la santé et la vertu, si bien que nulle part au monde on ne vit plus sainement qu'en Utopie. ${ }^{51}$ Et l'on retrouve donc ici le fameux mythe du "bon sauvage" incarné par l'Indien. Calquant leur organisation sur la nature, les indigènes peuvent se contenter de peu de lois. Connues de tous, elles rendent inutiles les avocats. ${ }^{52}$ Le rapport est ici évident entre ce naturel du "noble sauvage" et le droit naturel que l'on a tenté d'instituer dès le début de la Conquista. ${ }^{53}$ L'anecdote à propos de l'ambassadeur se veut révélatrice : couvert d'or, il suscite plus de dégoût que d'estime de la part des Utopiens car ceux-ci n'utilisent les métaux dits précieux que pour les chaînes des prisonniers, pots de chambre, etc. ${ }^{54}$ Or, il semble que les Indiens d'Amérique n'accordaient guère plus de valeur à l'or. 


\section{Las Casas et Thomas More : deux oeuvres parallèles}

L'Utopie présentait donc, d'une certaine façon, des solutions aux problèmes du Nouveau Monde. Il est probable d'ailleurs que More ait été au courant des négociations des Leyes de Burgos. Convoquée à la suite des protestations de Montesinos, l'assemblée s'était réunie pas moins de vingt fois en 1512. Puis ce fut au tour de Las Casas de faire scandale, en s'élevant, en 1515 et 1516 (soit au moment où More travaillait à son Utopie), contre les sévices infligés aux Indiens. Reçu par l'évêque Fonseca, il se heurta à une incompréhension totale et tenta d'approcher le roi. Toutefois, on peut raisonnablement penser que Las Casas n'attendit pas la mort de Ferdinand pour chercher auprès du futur Charles Quint une oreilie plus attentive.

Il rencontra donc le cardinal Cisneros et Adrien d'Utrecht, les régents, et leur soumit deux textes. Dans le premier, il dénonçait les abus des encomenderos ; dans le second, il ébauchait une nouvelle organisation des territoires du Nouveau-Monde. Las Casas était tellement convaincu du bien-fondé de sa requête que, dans son Historia de las Indias, il n'hésite pas à invoquer une prédestination inspirée par la Providence divine.

Parvenu à intéresser les autorités, Las Casas reçut le titre de Protecteur des Indiens et une allocation annuelle. Simultanément, Cisneros l'autorisa à expérimenter son organisation et à affranchir les Indiens qu'il jugerait capables. Cette première expérience échoua, mais d'autres aboutirent, et, surtout, Las Casas avait ouvert la voie. En 1550, Charles Quint rouvrit le débat sur les exactions des Espagnols en Amérique. A chaque occasion, Las Casas réapparut et dénonça les abus. Et c'est ainsi que fut créée la leyenda negra qui entoure les méfaits des colonisateurs du Nouveau-Monde. 55

Thomas More avait-il déjà entendu parler de Las Casas en 1515 ou 1516 ? C'est probable : la première intervention du futur Protecteur des Indiens; alors dans l'ombre de Montesinos, intervention qui devait conduire à la convocation de la Junta de Burgos, n'a pu manquer d'avoir un grand retentissement international, de même que les premières expériences de réorganisation, tentées dès 1516.

Aussi est-ce peut-être à Las Casas que pense More lorsqu'il mentionne dans sa préface un pieux, théologien de profession, qui souhaite évangéliser l'Utopie et envisage de se faire nommer par le pape évêque des Utopiens. Naturellement, cet homme pense que son ambition est justifiée car elle est dictée par une pieuse ferveur. ${ }^{56}$ Certes, à l'époque, Las 
Casas n'était pas encore évêque de Chiapa. Il n'avait pas même encore obtenu le mandat du Pape. Mais, il s'était déjà présenté aux autorités séculaires qui, elles, avaient reçu du Saint-Siège l'ordre de christianiser les nouveaux territoires.

Dans son Memorial de agravios hechos a los Indios, de 1516, Las Casas s'emploie à dénoncer les encomenderos qui tuent les Indiens à la tâche. ${ }^{57}$ Dans son Memorial de remedios para las Indias, toujours de 1516, il ébauche un plan détaillé pour une plus juste organisation du Nouveau Monde. ${ }^{58}$ Partant du principe que le système de l'encomienda doit être aboli car il concentre tous les pouvoirs entre les mains d'un seul homme, il le remplace par des comunidades composées d'une ville espagnole entourée de plusieurs villages indiens. Les mines et terres appartiennent à la communauté, qui y affecte ses Indiens.

Ce n'est pas un hasard si on a souvent vu dans les projets de Las Casas certains des éléments communistes qui caractérisent également l'Utopie de More. ${ }^{59}$ Le Protecteur des Indiens considérait que repos, nourriture et confort étaient indispensables pour que les travailleurs soient efficaces et jouissent d'une certaine dignité. Comme More, il accordait une grande importance aux prêtres, particulièrement en matière de droit, et à la construction d'églises et d'hôpitaux, à laquelle devaient travailler conjointement Espagnols et Indiens. Enfin, Las Casas entrait tellement dans les détails qu'il prévoyait une réglementation particulière pour chaque territoire et qu'il soumit même au Roi une note dans laquelle il décrivait les frais occasionnés par ses employés dans chacune des régions. ${ }^{60}$

Las Casas était plus proche de la réalité que More. Il se fondait sur sa propre expérience, tandis que l'auteur d'Utopie décrivait une communauté idéale. Toutefois, il y a de nombreux parallèles entre ces deux visions du Nouveau Monde. Et il est plus que probable que Thomas More avait connaissance, au moment où il ébauchait son ouvrage, des problèmes théologiques, juridiques et philosophiques qui avaient conduit aux Leyes de Burgos: on trouve dans Utopie, comme dans le Memorial de remedios, une antithèse idéale visant à refondre ce Nouveau Monde qui ne ressemble en rien à un paradis utopique.

\section{Conclusion}

Les dédicaces accompagnant l'Utopie ${ }^{61}$ suggèrent déjà une interprétation juridique de l'oeuvre. Ainsi, dans sa lettre envoyée à Thomas Lupset, Guillaume Budé tente d'expliquer l'ouvrage de More en faisant référence aux "jus naturale" et "jus gentium" de S. Thomas 
d'Aquin : le droit naturel permettrait au plus fort de tirer profit du droit positif pour exploiter son prochain. Or, Utopie est empreinte de l'amour que le chrétien se doit de porter à son prochain, ainsi que des principes d'égalité, de paix et d'indifférence à l'égard des biens terrestres. Et c'est précisément cela qui lui permet de s'extraire de la perpétuelle dichotomie droit civil / droit canon. ${ }^{62}$

Autre lettre intéressante, celle de Pierre Gilles à propos de la localisation d'Utopie, fait allusion à de nombreux pays récemment découverts. Et Gilles d'ironiser en signalant que tous ces pays sont aussi peu représentés sur les cartes qu'Utopie elle-même. ${ }^{63}$

Tout ceci démontre la vigueur des problèmes du Nouveau Monde à l'époque de More et suggère qu'il est bien question de l'Amérique dans l'Utopie. Bien sûr, le nouveau continent n'est pas le centre de l'Utopie, même si, plus tard, de nombreux textes sud-américains s'en sont inspirés. Mais on peut affirmer que l'ouvrage de More a eu de nombreux lecteurs en Amérique Latine et que, surtout, il est indirectement issu des conflits touchant le Nouveau Monde à l'époque.

On peut objecter que More avait déjà conçu l'Utopie durant son voyage en Flandres. Mais, à supposer qu'il n'était pas au courant de tous les problèmes du Nouveau Monde à ce moment-là, et que son beau-frère John Rastell n'avait pas réussi à éveiller son intérêt, il n'en est pas moins évident que Pierre Gilles lui a offert les moyens de s'initier à toutes ces questions.

Tout d'abord, l'Amérique était plus familière aux Flamands qu'elle ne l'était aux Anglais, de par la présence du futur Charles Quint. Et le Nouveau Monde occupait en Flandres les conversations des milieux diplomatiques et politiques. D'autre part, nombreux étaient les notables qui se retrouvaient chez Pierre Gilles, lequel, en outre, s'intéressait à l'histoire du droit. On trouvait chez lui Erasme, Busleiden et Vivès, par exemple, qui ont eu tout loisir de fournir à More des données sur la situation de l'Espagne.

La problématique du Nouveau Monde tournait autour du droit de prise de possession, du bien-fondé de la guerre, et de la cohabitation. Les textes légaux, réunis sous le nom de Leyes de Burgos, traitaient des droits des Indiens, de l'esclavage, de l'évangélisation, de l'éducation, de la répartition du travail, de l'affectation des indigènes et de l'organisation générale de l'encomienda. Simultanément, et en dépit de la cupidité de certains Conquistadors, renaissaient les mythes de l'Age d'Or et du Bon Sauvage. Tout cela n'a pu manquer d'inspirer les esprits fertiles tels que celui de More. 
Thomas More, dans son Utopie, propose des solutions à bon nombre de problèmes spécifiques du Nouveau Monde. Il y légitime la colonisation, l'emploi de la force armée, et y traite bien d'autres questions annexes. Comme les Rois espagnols, il se place dans la tradition aristotélicienne lorsqu'il parle de défense des frontières, de protection des alliés, de représailles. Il souligne la vanité des conflits religieux puisque les Utopiens sont gens tolérants et enclins à embrasser la religion chrétienne. Quant à l'esclavage, il ne fait que l'aborder en signalant qu'il est préférable à la liberté dans la misère. Il insiste sur la nécessité de bannir l'oisiveté et sur les bienfaits du travail dans la dignité. Enfin, il effleure la question de l'éducation en déclarant que les Utopiens sont particulièrement studieux et favorisés par leur mode de vie naturel.

Les projets et réalisations de Las Casas sont plus proches de la réalité sociale que ne l'est l'Utopie. Mais on peut raisonnablement penser que l'empressement du Protecteur des Indiens a eu des échos, qui sont parvenus jusqu'à Thomas More, et que celui-ci s'est inspiré indirectement du personnage pour décrire son "homme pieux" qui veut partir en Utopie évangéliser les peuplades indigènes.

Cette simultanéité et ces parallèles entre les oeuvres de Las Casas et More semblent donc bien indiquer que les premières n'étaient pas étrangères à la conception de l'Utopie. Il est impossible de faire la part des choses et de déterminer avec exactitude quels sont les emprunts à l'Antiquité et les inspirations purement contemporaines dans l'ouvrage de More. Mais, et c'est là le principal objet de notre recherche, nombreux sont les éléments de la complexe problématique du Nouveau Monde qui éclairent les fondements de 1'Utopie de Thomas More.

\section{Université de Cologne Albertus Magnus Platz Romanisches Seminar D $5000 \mathrm{Köln}$}
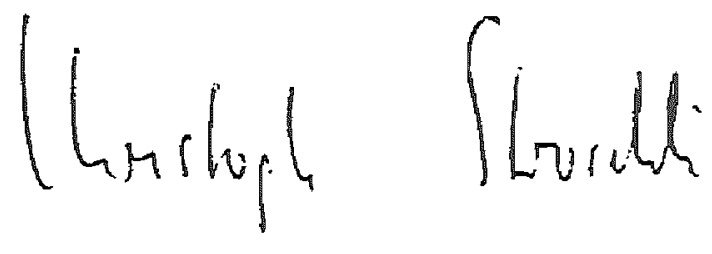

N O T E S

1. Cf. Wilhelm Voßkamp (éd.), Utopieforschung, Stuttgart 1982 (3 vol.); Hinrich Hudde, Rassegna sull'utopia come genere letterario, dans : Lectures. Quadrimestrale di studi su testi e tipologie culturali di espressione francese, 11, Dec. 1982, p. 11-29 et p. 277-296 ; pour l'Espagne, cf. : F. López Estrada, Tomás Moro y España, Madrid 1980. 
2. Cf. à ce sujet: Hinrich Hudde, Fernández de Lizardi : literarische Utopie an der Schwelle der Unabhängigkeit Mexikos (accompagné de remarques sur les utopies modernes d'Amérique latine), dans : Literaturwissenschaftliches Jahrbuch der Görresgesellschaft 27, 1986, p. 253-268.

3. Cf. Marcel Bataillon, Etudes sur Bartolomé de Las Casas, Paris 1965, p. 46 et suiv.

4. Cf. Silvio A. Zavala, La "Utopía" de Tomás Moro en la Nueva España y otros estudios, Mexico 1937, p. 4 et suiv. ; Silvio A. Zavala, L'utopie de Vasco de Quiroga, dans : Nouvelles du Mexique 45/47, 1966, p. 15-18; Rafael Aguayo Spencer (éd.), Don Vasco de Quiroga. Documentos, Mexico 1940.

5. Hinrich Hudde, Der "Jesuitenstaat" - eine verwirklichte Utopie ? Über eine alte Vorstellung und ihr Fortwirken bis in die Gegenwart, dans : Lateinamerika-Studien 14, München 1984 (Universität Erlangen-Nürnberg), p. 43-64 ; H. Hudde, Griechisches Ideal und südamerikanische Wirklichkeit. Zu José Manuel Peramás' Vergleich zwischen Platons Staatsschriften und dem "Jesuitenstaat" in Paraguay, dans : Iberoamérica. Historiasociedad-literatura, Homenaje a Gustav Siebenmann, vol. 1, J.M. López de Abiada et T. Heydenreich (éd.), München 1983 (Lateinamerika-Studien 13, 1, Univ, Erlangen-Nürnberg), p. $355-367$.

6. Cf. sur "l'Etat Jésuite" : Clovis Lugon, La république des Guaranis (1610 I768). Les Jésuites au pouvoir, Paris (Les Editions ouvrières) 1970 ; cf. sur l'importance de Thomas More : Philip Caraman, S.J., Ein verlorenes Paradies. Der Jestuitenstaat in Paraguay, München 1979 (trad. de l'anglais : New York 1976), p. 113.

7. Cf. sur la tradition du thème de l'Etat idéal avant Thomas More : Hubertus Schulte Herbrüggen, Utopie und Anti-Utopie. Von der Strukturanalyse zur Strukturtypologie, Bochum 1960 (Beiträge zur englischen Philologie 43), p. 37-49; cf. en ce qui concerne d'autres sources, le recueil d'interprétations : William Nelson (éd.), Twentieth Century Interpretations of "Utopia", Englewood Cliffs 1968 ; sur l'ouverture sur le monde de la ville d'Anvers, cf. H. Schulte Herbrüggen, Politik und Utopie : Thomas Morus, dans : Jahrbuch der Thomas-Morus-Geselischaft 1981, P. Berglar, H. Boventer, H. Schulte Herbrüggen (éd.), Düsseldorf 1982, p. 13-23, ici p. 16.

8. Dans ce contexte on a même défendu la thèse que l'Utopie de Thomas More est la description d'une République qui a existé : Arthur E. Morgan, Nowhere was Somewhere. How History Makes Utopias and How Utopias Make History, Chapel Hill $1946,1976^{2}$.

9. Cf. ci-dessous notes 21-33.

10. Cf. Hans Peter Heinrich, Thomas Morus, Reinbek 1984, p. 13-14.

11. Jerry Mermel, Preparations for a Politic Life : Sir Thomas More's Entry into the King's Service, dans : The Journal of Medieval and Renaissance Studies 7, 1977 , p. 53-66, ici p. 55 et suiv. ; cf. aussi : William Nelson, Thomas More, Grammarian and Orator, dans : PMLA 58, 1943, 1, p. 337-352, ici p. 348 et suiv. 
12. Cf. J.H. Hexter, "Utopia" and Its Historical Milieu, p. xxiii-cxxiv, dans : The Complete Works of St. Thomas More, Vol. 4, E. Surtz, S.J. et J.H. Hexter (éd.), New Haven \& London 1965, p. xxxi.

13. Cf. sur le déroulement de sa mission en Flandres: Sir Thomas More. Neue Briefe. Mit einer Einführung in die epistolographische Tradition, Hubertus Schulte Herbrüggen (éd.), Münster 1966 (Neue Beiträge zur englischen Philologie), p. 3-6.

14. Cf. H. Schulte Herbrüggen, F. Unterweg, U. Baumann, H.P. Heinrich, Thomas Morus (1477/78-1535). Humanist-Staatsmann-Märtyrer, Katalog zur Ausstellung in der Universitäts-Bibliothek Düsseldorf (25.10.85-4.1.86), p. 9.

15. H.P. Heinrich, Thomas Morus, p. 68 et suiv.

16. Il s'agit de l'oeuvre de Pierre Gilles: Summae sive argumenta legum diversorum imperatorum, ex corpore divi Theodosii, novellis divi Valentiani /.../, Lovanii 1517. Sur Pierre Gilles, cf. : M.A. Nauwelaerts, Un ami anversois de More et d'Erasme, Petrus Aegidius, dans : Moreana IV, 15/16, 1967, p. 83-96 ; cf. aussi : Biographie Nationale, L'Académie Royale des sciences, des lettres et des beaux-arts de Belgique (éd.), Bruxelles 1880, t. 7, p. $780-783$.

17. Charles Fantazzi, Vives, More and Erasmus, dans : Juan Luis Vives (Arbeitsgespräch in der Herzog August Bibliothek Wolfenbüttel vom 6.-8.11.1980), A. Buck (éd.), Hamburg 1981, p. 165-176, ici p. 165.

18. Sur Vivès cf. : Alain Guy, Vivès ou l'Humanisme engagé, Paris 1972 ; sur Croy cf. : Sir Thomas More. Neue Briefe, H. Schulte Herbrüggen (éd.), p. 3 ; sur Las Casas cf. notes 55-60.

19. Cf. Marcel Bataillon, Erasmo y España, Mexico, Madrid, Buenos Aires $1979^{2}$ (trad. du français 1937), p. 72-82.

20. Sur Busleiden cf. : Biographie nationale, Académie Royale des sciences, des lettres et des beaux-arts de Belgique, Bruxelles 1872, t. 3, p. 205-208; sur les lettres de Thomas More à Erasme cf. : Thomas Morus. Briefe der Freundschaft mit Erasmus, üb. H. Schulte Herbrüggen, München 1985.

21. Cf. Richard Konetzke, Süd- und Mittelamerika I. Die Indianerkulturen Altamerikas und die spanisch-portugiesische Kolonialherrschaft, Frankfurt a. M. 1965 (Fischer Weltgeschichte 22), p. 225.

22. Sur les dispositions contre la dispersion des agglomérations indiennes $\mathrm{cf}$. $\mathbb{R}$. Konetzke (éd.), Colección de Documentos para la Historia de la Formación Social de Hispanoamérica. 1493-1810, t. 1, Madrid 1953 (CSIC), p. 9 (Instrucción para el gobernador y los oficiales sobre el gobierno de las Indias, 1503), p. 19 (Instrucción a Don Diego Colón, 1509). Du Mariage cf. ibid., p. 12 et suiv. (Instrucción para el gobernador y los oficiales sobre el gobierno de las Indias, 1503). 
23. Cf. ibid., p. 3, 6, 7-8, 10.

24. Cf. R. Konetzke, Süd- und Mitlelamerika I, p. 34-39. 25. Cf. R. Konetzke, Colección de Documentos, p. 14-15, 31-33. 26. Ibid., p. 5. 27. Cf. ibid., p. 9-13.

28. Ibid., p. 20-22, 34-36.

29. Cf. Silvio Arturo Zavala, La encomiendo indiana, Mạdrid 1935, p. 16-17.

30. Cf. R. Konetzke, Colección de documentos, p. 38-57. 31. Ibid., p. 38. 32. Ibid., p. 39. 33. Cf. Silvio A. Zavala, La encomienda indiana, p. 16-20. 34. Cf. R. Konetzke, Süd- und Mittelamerika, p. 297.

35. Pedro Mártir de Anglería, Décadas del Nuevo Mundo, Edmundo O'Gorman (éd.), 2 vol., Mexico 1964, t. 1, p. 141. ("... Ils se contentent de si peu que, dans les régions où ils vivent, les champs surabondent plutôt qu'ils ne manquent à personne...')

36. Cf. José Antonio Maravall, El descubrimiento de América en la Historia del pensamiento político, dans : Revista de Estudios Políticos 63, 1952, p. 244 ; cf. aussi sur la dimension utopique de la découverte de l'Amérique : José Luis Abellán, Historia crítica del pensamiento espafiol, t. 2, La edad de oro, Madrid 1979, p. 373-389.

37. Thomas More, The Complete Works, Vol. 4, Utopia, E. Surtz, S.J. et J.H. Hexter (éd.), New Haven et London 1965, p. 110-113.

38. Ibid., p. 98-103. 39. Ibid., p. 136 et suiv. suiv. 41. Ibid.,p. 198-217. 42. Ibid., p. 216-223. et suiv. $\quad 44$. Ibid., p. 114 et suiv., 122-125, 194 et suiv. suiv. 46. Ibid., p. 226-237. 47. Ibid., p. 180-185. et suiv., 124-129. 49. Ibid., p. 136-141, 184-187. 144-147. 51. Ibid., p. 160-179. 52. Ibid., p. 194 et suiv.

53. Cf. J.L. Abellán, Historia crítica del pensamiento español, p. 408 et suiv., cf. aussi Joseph Höffner, Kolonialismus und Evangelium, Spanische Kolonialethik im Goldenen Zeitalter, Trier $1969^{2}$, p. 59.

54. Thomas More, The Complete Works, Vol. 4, p. 150-157.

55. Cf. Pierre Mesnard, Bartolomé de Las Casas (1474-1566) à travers son quatrième Centenaire, dans : Moreana IV, 15/16, 1967, p. 401-430, en particulier p. 409-410; cf. aussi Ángel Losada, Fray Bartolomé de Las Casas a la luz de la moderna crítica histórica, Madrid 1970, pour la Junta de Valladolid en particulier p. 246 et suiv.

56. Thomas More, The Complete Works, Vol. 4, p. 42 et suiv.

57. Las Casas pense que deux raisons principales sont la cause de l'extermination des Indiens : "La primera, e] trabajo demasiado que a los Indios se ha dado por la mucha codicia de los que de España iban ; la segunda, tratarlos mal, no teniendo cuidado de les dar de comer ni vestir, como habían menester, según el trabajo pasaban". Fray Bartolomé de Las Casas, Opúsculos, cartas y memoriales, J. Pérez de Tudela Bueso (éd.), dans : Obras escogidas V, Madrid 1958 (BAE) p. 5. 
58. Cf. A. Losada, Fray Bartolomé de Las Casas, p. 105.

59. "Que vuestra señoría mande hacer una comunidad en cada villa y ciudad de los españoles, en que ningún tenga Indios conocidos ni sefîalados, sino que todos los repartimientos estén juntos y que hagan labranzas juntos, y los que hubieren de coger oro lo cojan juntos." B. de Las Casas, Opuisculos, cartas y memoriales, p. 6, cf. aussi Henry Raup Wagner, The Life and Writings of Bartolomé de Las Casas, Albuquerque 1967, p. 20.

60. Cf. "Memorial de remedios para las Indias" : B. de Las Casas, Opúsculos, cartas y memoriales, p. 5-27, ici p. 24 et suiv.

61. Cf. H. Süssmuth, Studien zur "Utopia" des Thomas Morus. Ein Beitrag zur Geistesgeschichte des 16. Jahrhunderts, Münster 1967, p. 139 et suiv.

62. Cf. la lettre de Guillaume Budé dans : André Prévost, L'Utopie de Thomas More, Paris 1978, p. 320 et suiv. ; W. Voßkamp va jusqu'à concevoir le symbole de construction juridique comme étant une offre symbolique "constructive" dans laquelle "der ökonomische bzw. juristische Diskurs den theologischen ablöst." Wilhelm Voßkamp, Thomas Morus' "Utopia" : Zur Konstituierung eines gattungsgeschichtlichen Prototyps, dans : W. Voßkamp (éd.), Utopieforschung, t. 2, Stuttgart 1982, p. 190.

63. Cf. la lettre dans: A. Prévost, L'Utopie de Thomas More, p. 340-341.

\section{Résumé}

La problématique du Nouveau Monde tournait autour du droit de prise de possession, du bien-fondé de la guerre, et de la cohabitation. Les textes légaux, réunis sous le nom de Leyes de Burgos, traitaient des droits des Indiens, de l'esclavage, de l'évangélisation, de l'éducation, de la répartition du travail, de l'affectation des indigènes et de l'organisation générale de l'encomienda. Simultanément, et en dépit de la cupidité de certains Conquistadors, renaissaient les mythes de l'Age d'Or et du Bon Sauvage. Tout cela n'a pu manquer d'inspirer More, d'autant plus qu'il avait passé un certain temps en Flandres où le Nouveau Monde occupait les conversations des milieux diplomatiques et politiques.

\section{Précis}

The problematics of the New World centred on the law of taking possession, the grounds for a just war, and cohabitation. The legal texts, collected under the title of Leyes de Burgos, dealt with the rights of the Indians, slavery, evangelisation, education, the distribution of labor, the assigning of the natives and the general organisation of the encomienda. At the same time, and despite the greed of certain Conquistadors, the myths of the Golden Age and the Noble Savage were 
being revived. All this cannot have failed to inspire More, especially as he had spent some time in Flanders where the New World was much spoken of in diplomatic and political circles.

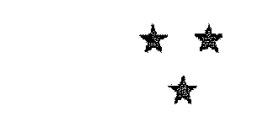

\section{UTOPIAN STUDIES}

Journal of the Society for Utopian Studies

Editor : Lyman Tower Sargent, Political Science, University of Missouri-St Louis Associate Editor : Lise Liebacher-Ouvrard, French, University of Arizona

Managing Editor : Stacey Tipp, Political Science, University of Missouri-St Louis Editorial Board:

Carol Kolmerten, English and American Studies, Hood College ; Susan Matarese, Political Science, University of Louisville ;

Nicholas D. Smith, Philosophy, Virginia Polytechnic Institute and State University.

Utopian Studies, a new journal, is the official journal of the Society for Utopian Studies, an interdisciplinary, international association founded in 1975. Utopian Studies, which is published twice yearly, replaces a series of the refereed proceedings of the annual meetings which has been published for four years. Utopian Studies incorporates Utopus Discovered, the Newsletter of the Society for Utopian Studies, which has been published for fourteen years.

Utopian Studies publishes articles in English and arrangements have been made to publish English translations of articles submitted in French, German, and Italian. Utopian Studies will publish a substantial number of book reviews. Thus, Utopian Studies provides an international, interdisciplinary forum that the field of utopian studies previously lacked.

Submissions, inquiries, and books for review should be sent to the Editor at the following address : Utopian Studies, Department of Political Science, University of Missouri-St. Louis, St. Louis, MO 63121-4499.

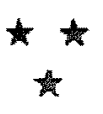

